\title{
EL USO DE LA AUTODIRECCIÓN EN EL CONTROL DEL PROCESO TERAPÉUTICO EN UN CASO DE ESQUIZOFRENIA
}

\author{
Anisia Méndez \\ CUMEN, Centro Terapéutico. Santa Cruz de Tenerife
}

\section{RESUMEN}

En este estudio se presenta el tratamiento psicológico de un caso de esquizofrenia paranoide. El análisis inicial del paciente pudo constatar una capacidad intelectiva adecuada, preponderancia de sintomatología positiva y elevada conciencia de enfermedad. El tratamiento consistió en el uso de la reestructuración cognitiva (creencias irracionales asociadas a los delirios), la exposición en vivo a estímulos temidos y entrenamiento en habilidades sociales $y$ de resolución de problemas. Debido a las caracteristicas del paciente, el proceso terapéutico fue autodirigido por medio de un modelo de autorregistro que favorecía la toma de decisiones por parte del paciente y el control de su propia evolución. Los resultados mostraron una reducción en los comportamientos-objetivos: pensamientos negativos disfuncionales, conductas evitativas y de comprobación. Esa reducción se pudo constatar tanto en la evaluación post-tratamiento como en el seguimiento. Asimismo disminuyó la sintomatología positiva, el nivel de depresión, las conductas evitativas de carácter fóbico, la psicopatología general

Correspondencia: Anisia Méndez Izquierdo, CUMEN Centro Terapéutico, C/. Juan Pablo II, 21, oficina C, 38004, Santa Cruz (Santa Cruz de Tenerife), C-e: ctcumen @telefonica.net 
y aumentaron sus habilidades sociales. Estos resultados se discuten de acuerdo con la idoneidad del uso de la autodirección en algunos casos de psicosis en los que se pueda observar la presencia de síntomas favorecedores para la autorregulación.

Palabras clave: ESQUIZOFRENIA PARANOIDE, TRATAMIENTO COGNITVO-CONDUCTUAL, AUTORREGULACIÓN.

\section{SUMMARY}

This paper presents the psychological treatment of a case of paranoid schizophrenia. The initial analysis of the patient could state an appreciable intelectual ability, prevalence of positive symptomatology and high conscience of disease. The treatment consisted of the use of the cognitive restructuring (irrational beliefs associated with the deliriums), in vivo exposure to phobic stimuli, and training in social skills and problems-solving. Due to the characteristics of the patient, the therapeutic process was selfguided by means of a model of self-observation that was favoring decisions taking and to control his progress. The results showed a reduction in the therapy targets: negative disfunctional thoughts, avoidance behaviors and checking behaviors. This reduction could be verified both at post-treament phase and at follow-up. Besides, a decrease was also observed in positive symptoms, the level of depression, the avoidance-phobic cognitions, the general psychopathology symptoms, and an increase in his social skills. These results are discussed according to the adapted use of the self-direction in some cases of psychosis, when the presence of favouring symptoms for self-regulation can be observed.

Key words: PARANOID SCHIZOPHRENIA, COGNITIVE-BEHAVIORAL TREATMENT, SELF-REGULATON.

\section{INTRODUCCIÓN}

Los principales avances en las estrategias de tratamiento para la esquizofrenia se han basado en el modelo de vulnerabilidad-estrés 
(Zubin y Spring, 1977). Este modelo postula una interacción entre estresores ambientales y elementos de vulnerabilidad biológica para producir la aparición de una crisis y las incapacidades psicosociales secundarias características de la esquizofrenia. Esta propuesta ha propiciado la aparición de una gama de aproximaciones terapéuticas fundamentadas en la eliminación o reducción de los dos polos que la componen: los estresores ambientales y la vulnerabilidad biológica subyacente. Entre ellas, se pueden destacar las intervenciones familiares psicoeducativas, el entrenamiento en habilidades sociales, los tratamientos cognitivo-conductuales y los paquetes integrados multimodales (Birchwood, Hallet y Preston, 1989; Wykes, Tarrier y Lewis, 1998).

En relación a las intervenciones familiares psicoeducativas, la desinstitucionalización de los pacientes durante las dos últimas décadas favoreció la incorporación de los familiares como un importante recurso terapéutico, fundamentalmente a partir de las investigaciones realizadas sobre un contexto familiar patologizante, denominado emoción expresada. Todo ello ha dado lugar a distintos modelos terapéuticos de intervención familiar, entre los que podemos citar el paquete de intervenciones sociofamiliares de Leff (Kuipers, Leff y Lam, 1992; Leff, Kuipers, Berkowitz y Sturgeon, 1985); el modelo psicoeducativo de Anderson (Anderson, Reiss y Hogarty 1986); las intervenciones cognitivo-conductuales (Barrowclough y Tarrier, 1992; Tarrier y Barrowclough, 1995); y la terapia conductual familiar (Falloon, Laporta y Fadden,1993). Globalmente, estos modelos psicoeducativosconductuales manifiestan una considerable variación en la importancia que se le concede a cada uno de los componentes del tratamiento y grado de estructuración del mismo. Sin embargo, comparten una aproximación positiva y una relación de trabajo genuina con los familiares. En relación a la eficacia de las intervenciones familiares, en general se ha observado que, combinadas con medicación antipsicótica, han resultado ser eficaces en la reducción de la carga y la emoción expresada en los familiares, la sintomatología clínica, las recaídas y rehospitalizaciones de los pacientes, así como en su rentabilidad económica para los servicios sanitarios (Goldstein, Rodnick, Evans, Philip, MayPRA y Steinberg, 1978; Goldstein, 1994; Liberman, 1986; Perris y Skagerling, 1994; Tarrier, Beckett y Harwood, 1993; Kingdon 
y Turkington, 1991; Camisa, Bockbrader y Lysaker, 2005; Freeman, Garety y Kuipers, 2002; Garety, Fowler y Kuipers, 2000; Heissen, Liberman y Kopelowicz, 2000; Jolley, Garety y Craig, 2003; Joyce, Leese y Kuipers, 2003; Kingdon, 2004; Kingdon y Turkington, 2005; Kuipers, 2005; Pilling, Bebbington y Kuipers, 2002; Pilling, Bebbington y Kuipers, 2002; Rathod, Kingdon y Smith, 2005; Slade, Holloway y Kuipers, 2003; Smith, 2005; Stevens y Sin, 2005; Stolar, 2005; Szmukler, Kuipers y Joyce, 2003; Tarrier, Kinney y McCarthy, 2001). Como aspecto crítico cabe decir que, a pesar de la evidencia empírica de eficacia de estos programas, resulta llamativa la resistencia que algunos familiares ponen para su participación.

Con respecto al entrenamiento en habilidades sociales en la esquizofrenia, se fundamenta en que las relaciones interpersonales conforman un elemento central para lograr un adecuado desempeño y un factor determinante para la integración social y adaptación de la persona a largo plazo. La esquizofrenia es un trastorno que incluye, como uno de sus rasgos distintivos, el déficit en el funcionamiento social e interpersonal, una fuente de estrés que puede contribuir a las recaídas y exacerbaciones sintomáticas (Bellack, Morrison y Mueser, 1989; Liberman, 1993). Este déficit ha demostrado ser relativamente estable a lo largo del tiempo (Smith, Bellack y Liberman, 1996a), y está altamente relacionado con la sintomatología negativa (Heinssen, Liberman y Kopelowicz, 2000). Por ello, a lo largo de las últimas dos décadas, el entrenamiento en habilidades sociales ha representado una técnica central para potenciar ese pobre funcionamiento social. Como ocurre con las terapias familiares, este entrenamiento ofrece cierta variabilidad (Liberman, 1986). Corrigan (1991) en un meta-análisis de 73 estudios con diferentes patologías, concluyó que los pacientes que participaban en este tipo de programas ampliaban su repertorio de destrezas, las mantenían durante varios meses después de la finalización del tratamiento y manifestaban una disminución de la sintomatología relacionada con los déficit a nivel social. Siendo un método más útil para pacientes ambulatorios que para pacientes internos. Benton y Schroeder (1990), en otro metaanálisis de 27 estudios, sólo con población con esquizofrenia, concluyeron que se produce un impacto positivo sobre medidas conductuales de dichas habilidades, sobre la asertividad autoevaluada 
y sobre la tasa de altas hospitalarias. El impacto sobre la recaída, sin embargo, era más moderado, y ni la homogeneidad del diagnóstico, el número de técnicas empleadas o la cantidad de entrenamiento, parecen diferenciar significativamente los resultados.

En referencia a los tratamientos cognitivo-conductuales, éstos se han utilizado básicamente para el tratamiento de los delirios y las alucinaciones ante la constatación de que los pacientes desarrollan sus propias estrategias de afrontamiento para minimizar la frecuencia, gravedad y/o exacerbación de los síntomas psicóticos (Norman y Townsend, 1999). La terapia cognitiva ha aportado técnicas de apoyo a los pacientes con el objetivo de fomentar el manejo de sus experiencias psicóticas, enseñándoles no sólo a re-etiquetar sus creencias delirantes sino, también, a dar sentido psicológico a sus sintomas (Yusupoff y Tarrier, 1996). En general, los hallazgos más consistentes en relación a la eficacia de las intervenciones cognitivo-conductuales indican beneficios significativos en la reducción de la gravedad y cantidad de los síntomas psicóticos, especialmente en los positivos (Drury, Birchwood y Cochrane, 1996a; Haddock y Tarrier, 1998; Jakes, Rhodes y Turner, 1999; Kuipers, Garety y Fowler, 1997; Tarrier, Becket y Harward, 1993a; Tarrier, Barrowclough y Haddock, 1999); en la mejora en el cumplimiento del régimen farmacológico (Kemp, David y Hayward, 1996a; Kemp, Hayward, Applewhaite, Everit y David, 1996b; Kemp, Kirov y Everit, 1998); en la disminución del número de recaídas (Haddock, Tarrier y Morrison, 1999); y de síntomas residuales en las recaídas (Drury, Birchwood y Cochrane, 1996b).

Por último, con respecto a los paquetes integrados multimodales, este acercamiento parte del supuesto de que las personas que padecen esquizofrenia manifiestan deficiencias en distintos niveles de funcionamiento de organización de la conducta (nivel atencionalperceptivo, nivel cognitivo, nivel micro-social y nivel macro-social); que las deficiencias de un nivel repercuten negativamente en el funcionamiento del resto de niveles; asimismo, los diferentes niveles pueden guardar una relación jerárquica entre sí. Brenner, Hirsbrunner y Heimberg, (1996) desarrollaron un modelo que sivvió de fundamento para el desarrollo de la psicoterapia integrada para la esquizofrenia o terapia psicológica integrada (IPT). Ésta es un programa de intervención grupal, cuyo objetivo básico es la mejora de las habilidades 
cognitivas y sociales de personas con esquizofrenia, que se ha aplicado a más de 700 pacientes de diferentes países. Consta de cinco subprogramas ordenados jerárquicamente, de tal forma que las primeras intervenciones están dirigidas a las habilidades cognitivas básicas, las intermedias a la transformación de las habilidades cognitivas en respuestas verbales y sociales, y las últimas, al entrenamiento de los pacientes en la resolución de problemas interpersonales más complejos. Lo estudios evaluados con IPT indican, de forma general, que el subprograma inicial de la Diferenciación Cognitiva mejora los procesos cognoscitivos elementales de los pacientes, tales como la atención, abstracción y formación de conceptos, aunque el desempeño sigue estando por debajo del rango normal. Por lo que paradójicamente, y a pesar del reconocimiento de la multifactorialidad causal que tiene la esquizofrenia y, por tanto, de su abordaje multidimensional, los modelos de tratamiento integrados han sido muy poco utilizados. Esta carencia está siendo revisada, y actualmente existe una creciente unanimidad de criterio en torno a la necesidad de integrar los diversos procedimientos terapéuticos en programas generales que abarquen, de un modo global, las distintas y permanentes necesidades que presentan las personas con trastornos mentales crónicos entre ellos, la esquizofrenia (Chambon, Marie-Cardine y Dazord, 1996; Haddock y Slade, 1996; Lehman, Carpenter, Goldman y Steinwachs, 1995; Liberman, 1993; Liberman, Vaccaro y Corrigan, 1995; Penn y Mueser, 1996).

En general, los programas de intervención llevados a cabo comparten elementos comunes en relación a dos ámbitos. Por un lado, la necesidad de combinar dichos programas con la medicación antipsicótica y, por otro, la necesídad de protocolizar y sistematizar la intervención, en la medida en que los pacientes no tienen una buena conciencia de su trastorno, poseen una baja capacidad de autoanálisis y no participan activamente o no están especialmente motivados para ello (síntomas negativos).

Esto ha hecho que el abordaje psicoterapéutico posea una metacaracteristica que no es usual en el abordaje en otros trastornos: la dirección activa por parte del terapeuta como guía de procedimiento terapéutico a aplicar. Usualmente, se reclama una participación activa de los pacientes, sin embargo, en las personas con esquizofrenia, el 
terapeuta tiene que asumir un papel más activo y dirigista ante la necesidad de que el paciente lleve a cabo las prescripciones psicoterapéuticas. Una posición de menor control puede hacer que decaigan tanto la adherencia como su rigor.

Sin embargo, las ventajas de una participación más activa del paciente, facilitando su auto-regulación son evidentes. ¿Existen condiciones en la esquizofrenia en las que se pueda dar esa mayor participación? La conciencia del estado mental puede estar perfectamente clara en algunos pacientes, pueden tener una buena capacidad de autoanálisis y pueden reclamar una mayor participación en la toma de decisiones terapéuticas.

A continuación presentamos un caso en el que consideramos que se dieron las condiciones adecuadas para que un paciente, con esquizofrenia paranoide, fuera agente activo de su propio cambio, corresponsabilizándose de su auto-regulación en la puesta en marcha del programa cognitivo-conductual aplicado.

\section{MÉTODO}

\section{Participante}

Paciente varón, de 28 años de edad, que acude a consulta por la creencia de presentar dificultades para distinguir lo que es realidad de lo que es ficción y porque se considera inmaduro.

De la entrevista inicial de anamnesis se pueden destacar los siguientes datos: referidos al ámbito familiar, relata que es el menor de cuatro hermanos varones con los que mantiene unas buenas relaciones. Sus padres están jubilados. Define al padre como autoritario, posesivo y dominante con el que mantiene, en el momento de la entrevista, una relación de dependencia debido a un comportamiento sobreprotector. Define a la madre como insegura. El paciente destaca como sucesos vitales remotos problemas físicos de la madre, los castigos recibidos, y un antecedente familiar con diagnóstico de esquizofrenia. Como sucesos vitales estresantes recientes expone el cambio de lugar de residencia, mostrando elevadas dificultades de adaptación, y el fallecimiento de un buen amigo del paciente quien, a su vez, habia sido diagnosticado de esquizofrenia. Profundizando en 
cómo valora el paciente su infancia y adolescencia, manifiesta una visión global positiva de tal periodo, a excepción de su miedo intenso a los insectos, creyendo tener escorpiones en su ropa interior, la precaria salud física de su madre, viviendo tal situación como una experiencia de abandono por parte de aquella, y frecuentes y repetidas humillaciones, por parte de su profesora y compañeros de clase. Con respecto al ámbito académico-laboral del paciente, refiere haber cursado estudios hasta COU para posteriormente, realizar diversos cursos relacionados con la informática y durante el transcurso del año 2002 cursar un módulo de F.P. de Técnico Sanitario, que ha finalizado en la actualidad. El paciente refiere que le genera un gran bienestar y satisfacción ayudar a personas de edad avanzada colaborando por ello, y de forma voluntaria, en diferentes asociaciones durante un periodo aproximado de tres años. En esa época el paciente manifestaba alucinaciones de tipo visual y auditivo lo que provocaba y precipitaba el abandono de la colaboración con tales asociaciones. Por último, y en relación al ámbito social, el paciente presenta una escasa red de apoyo social fundamentándose exclusivamente en el contacto frecuente con uno de sus hermanos.

El paciente refiere como motivo de consulta, apreciando una forma de expresión verbal atropellada y circunstancial, su percepción de presentar un fuerte bloqueo emocional, dificultad a la hora de la toma de decisiones e imposibilidad de expresar externamente sus emociones, una intensa preocupación al creer no saber diferenciar "lo que es real de lo que no", iniciándose estos síntomas en torno a los 18 años de edad.

Hasta el momento había sido tratado, y como única forma de abordaje terapéutico, con técnicas de relajación, manifestando que no le había generado ningún beneficio. Una semana antes de acudir a nuestra consulta, visitó un Centro Hospitalario en donde se le diagnosticó "ansiedad crónica" recetándole, como tratamiento único, Alprazolam. Dado la inestabilidad del diagnóstico del paciente y los síntomas referidos por él mismo, se decide la derivación a una especialista en psiquiatría. Este profesional le diagnostica esquizofrenia paranoide (OMS; CIE-10: F20.0) y le prescribe un tratamiento farmacológico adecuado al mismo (Olanzapina). 


\section{Evaluación}

La evaluación consistió en la aplicación y cumplimentación de una batería de pruebas, en tres momentos temporales: en la fase inicial pretratamiento, en la $12^{\mathrm{a}}$ sesión, inmediatamente después del tratamiento (post-intervención) y doce meses después (seguimiento). A continuación, se expone un breve resumen de la batería de pruebas empleadas, justificando su uso y resultados obtenidos, tras la primera sesión de evaluación, de la manera que sigue:

"Escala para el Síndrome Positivo y Negativo de la Esquizofrenia" (The Positive and Negative Sindrome Scale (PANSS), Kay, Fiszbein y Opler, 1987). Esta escala consta de 30 ítems, que a su vez conforman cuatro escalas: escala positiva (PANSS-P), escala negativa (PANSS-N), escala compuesta (PANSS-C) y psicopatología general (PANSS-PG). Cada uno de los ítems que conforman la escala, se puntúa de acuerdo a una escala de 7 puntos tipo Likert de intensidad y gravedad de la sintomatología. Después de una entrevista semiestructurada, es cumplimentada por un clínico entrenado al efecto. Se empleó esta escala tanto, para confirmar el diagnóstico como, para el establecimiento de la línea de base con el fin de contrastar los efectos del tratamiento. Del primer pase de dicha escala se obtuvo para la escala positiva el percentil 50 , para la escala negativa el percentil 25 y para la escala de psicopatología general el percentil 50. De todo ello se concluye, inicialmente, que el paciente manifestaba una marcada sintomatología positiva y psicopatología general (destacando dentro de ésta, la preocupación somática, evitación social activa y sintomatologia mixta ansiosa-depresiva) y la existencia, aunque en menor medida que las anteriores, de sintomatología negativa.

"Inventario de Depresión de Beck" (Beck Depresión Inventory, BDI; Beck, Ward, Mendelson, Mock y Erbaugh, 1961). Se decidió por el empleo de este instrumento para rastrear la posible sintomatología depresiva, detectada en la entrevista inicial y en la PANSS. El inventario consta de 21 ítems que evalúan fundamentalmente, los síntomas clínicos de la depresión. Tras la cumplimentación del paciente de este inventario, se obtuvo una puntuación directa de 37 de lo que se extrae que el paciente manifestaba, en los momentos iniciales del tratamiento, un nivel de depresión grave. 
"Inventario de Agorafobia" (Echeburúa, Corral, García, Paez y Borda, 1992). Este cuestionario consta de 69 ítems, estructurados en dos partes. La primera evalúa, mediante una escala tipo Likert de 0 a 5 puntos, distintos tipos de respuestas alteradas (motoras, psicofisiológicas subjetivas y cognitivas) del paciente, solo y en compañía, ante las situaciones estimulares más habituales. La segunda parte, mide la variabilidad de las respuestas en función de los factores que contribuyen a aumentar y reducir la ansiedad. Se escogió este instrumento por la necesidad de profundizar en los diferentes estímulos fóbicos que ocasionaban las diferentes conductas de evitación / escape, especialmente en el mundo social, y para complementar la información necesaria para realizar una análisis adecuado del caso. De los resultados se extrae, para la escala de respuestas motoras una puntuación directa de 200 (destacando, la presencia de las calificaciones más elevadas en los ítems relacionados con las situaciones sociales), para la escala de respuestas psicofisiológicas una puntuación directa de 90 y por último, para la escala de respuestas cognitivas un puntuación directa de 51. Con todo ello, se concluye que el paciente manifiesta una marcada sintomatología en relación a las tres escalas de las que consta este cuestionario.

"Cuestionario de Habilidades Sociales" (Segura, 1981). Este instrumento consta de veinte situaciones diferentes que evalúan distintas dimensiones del comportamiento interpersonal y de asersión (presentarse, participar en las conversaciones superficiales, hablar de temas más íntimos, expresar desacuerdos, etc.). Se elige entre cuatro formas diferentes de actuación (adecuada, con ansiedad, inhibición e inhabilidad). Se utilizó este instrumento como parte del establecimiento de la línea de base de uno de los motivos referidos por el paciente y que hacía hincapié en la incapacidad percibida de expresar emociones y de relacionarse eficazmente con los demás, plasmada en sus comportamientos de evitación social activa que manifestaba en la primera valoración realizada mediante la PANSS, dentro de la escala de psicopatología general de la misma. De la cumplimentación del paciente de este instrumento se concluye que manifiesta, en la mayor parte de las dimensiones, tanto una inhibición como inhabilidad de tales comportamientos.

"Protocolos de autorregistro". Se elaboraron distintos modelos de 
autorregistro para el paciente. Inicialmente se le solicitó que se autorregistrara de acuerdo con un protocolo 'situación, siento, pienso, hago' con la finalidad de establecer enlaces funcionales entre sus comportamientos y pensamientos desadaptativos y captar las principales creencias irracionales, que ocasionaban un elevado grado de malestar al paciente y que limitaban su vida diaria. Después, se utilizaron otros protocolos en función del desarrollo terapéutico y la autorregulación del paciente. Estos protocolos de ilustran más adelante.

Una vez expuestos todos los instrumentos empleados para la elaboración de un análisis del caso sobre el que se fundamentará el establecimiento de los objetivos terapéuticos, se puede constatar que los estímulos externos que al paciente le generan un mayor grado de malestar, destacar: la posibilidad de establecimiento de relaciones interpersonales, el contacto con insectos, afrontar la toma de decisiones y solución de problemas, hablar en público, la posibilidad de cometer errores, mirar a la gente a la cara directamente, pasear por los alrededores de su casa y la oscuridad y por otro, a los estimulos internos, pensamientos recurrentes (rumiativos) y anticipatorios de sentirse constantemente vigilado - amenazado y la posibilidad de morirse, tales como: "Iban en contra de mî", "los demás me están escuchando", "rnientras duermo, pienso que me están mirando", "cuatro personas van por la calle insultándome y persiguiéndome", etc...

De todo lo extraído hasta el momento, y perfilando el análisis del caso, nos planteamos la hipótesis consistente en que la vulnerabilidad existente en el paciente a raíz de sus antecedentes familiares (familiar cercano con esquizofrenia) se ve exacerbada por una serie de estresares ambientales, que actúan como sucesos vitales estresantes, destacando: problemas de relación interpersonal con sus compañeros de clase, respuestas verbales inadecuadas por parte de su profesora en el colegio, salud precaria de la madre, comportamientos desadaptativos por parte del padre y fallecimiento reciente de un buen amigo suyo, y que en combinación con dicha vulnerabilidad precipitan el inicio de la sintomatología propia de la esquizofrenia.

De lo expuesto hasta aqui, se extraen las siguientes conductas problemas y los objetivos terapéuticos sobre los que se sustentará la terapia a realizar (ver tabla 1 ). 
Tabla 1.- Conductas problema del caso y objetivos terapéuticos

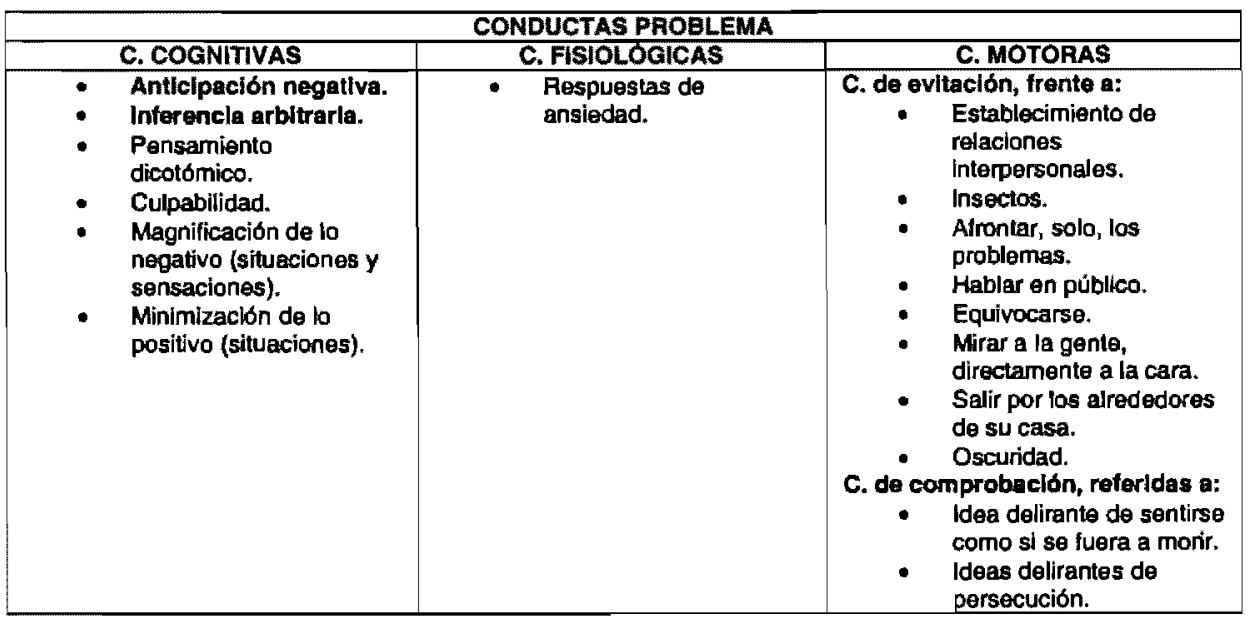

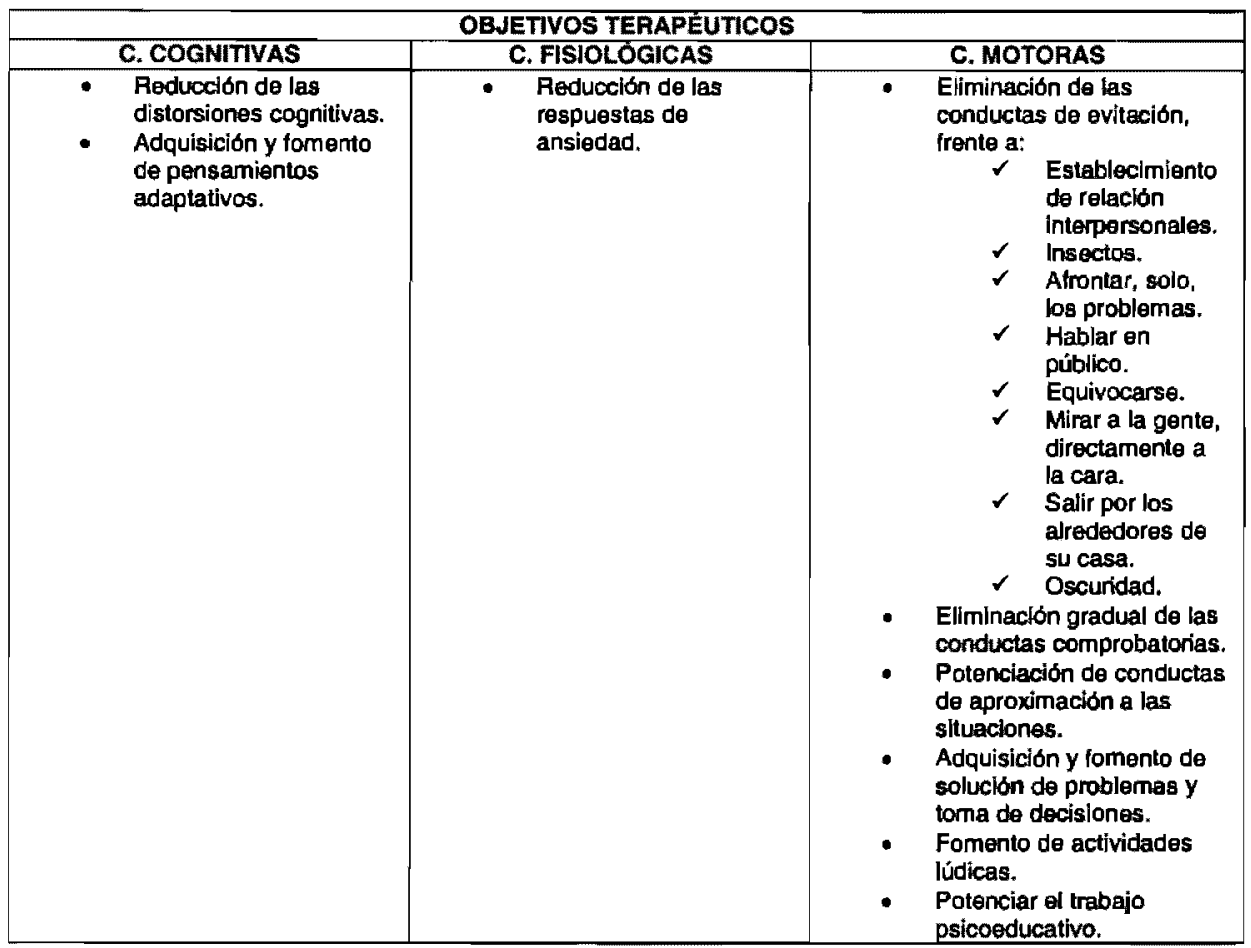




\section{PROCEDIMIENTO}

Se presenta a continuación (tabla 2), el cronograma y los objetivos terapéuticos de cada una de las sesiones, que se llevaron a cabo para cada una de las fases de las que constó la intervención global del caso.

Tabla 2.- Objetivos terapéuticos especificos para cada bloque de sesiones del tratamiento del caso

\begin{tabular}{|c|c|}
\hline NÚMERO DE SESIONES & OBJETIVOS TERAPÉUTICOS GLOBALES \\
\hline Tres (1*a la $3^{x}$ sesión) & Evaluación global del paciente \\
\hline Dos (4 y 5" sesión) & Psicoeducación del paciente y familiares. \\
\hline Slete (6! a $12^{\prime}$ seslón) & $\begin{array}{l}\text { Desarrollo y prófundización de la reestrucluración cognitiva (de una } \\
\text { manera activa) y exposición en vivo a los estímulos externos } \\
\text { temidas (fóbicos). }\end{array}$ \\
\hline Seslones de seguimlento & $\begin{array}{l}\text { Alianzamiento de las técnicas aplicadas y desarrolladas durante la } \\
\text { fase de intervención (reestructuración cogniliva y exposición en } \\
\text { vivo). potenciación de la rehabilitación social del paciente, } \\
\text { afianzamiento de las mejorías alcanzadas hasta el momento y } \\
\text { seguimiento. }\end{array}$ \\
\hline
\end{tabular}

Como se puede apreciar en la tabla anterior, durante las tres primeras sesiones se obtuvo la evaluación global. Durante el transcurso de las dos sesiones siguientes se profundizó en la potenciación del conocimiento por parte de los familiares del paciente, que acudieron a las mismas de forma voluntaria, de los aspectos básicos y definitorios de la patología de su familiar (esquizofrenia paranoide). Se resolvían aquellas dudas que pudieran presentar a la hora del manejo de las situaciones conflictivas y la reducción de los niveles de estrés e incertidumbre que presentaban dichos familiares en un primer momento.

A lo largo de las siete sesiones siguientes se pretendía, por un lado, en referencia a los estímulos externos, potenciar el empleo de la técnica de la exposición gradual en vivo a los estímulos temidos y el fomento de habilidades de comunicación interpersonal.

Por otro lado, con respecto a los objetivos terapéuticos planteados en relación a los estímulos internos por el paciente, se potenció el aprendizaje y la práctica de la reestructuración cognitiva bajo un enfoque en el que se daba prioridad y especial importancia al encuentro 
conjunto, paciente y terapeuta, del mejor modelo de autorregistro para la re-etiquetación de las creencias delirantes, entendiendo como mejor modelo de autorregistro, aquél que el propio paciente refiriera como el más adecuado, práctico y de fácil cumplimentación. De esta forma, se le hacía más asequible el manejo de sus pensamientos y creencias irracionales, favoreciendo con todo ello, la aceptación de la modificación de los mismos.

La progresión de los modelos de autorregistros empleados hasta la consecución del definitivo y que resultó eficaz en la mejoría del paciente en la minimización de sus creencias delirantes, se recogen en la tabla 3 , incluyendo algunos comentarios del propio paciente.

Tabla 3.- Diferentes modelos de autorregistros, utilizados por el paciente.

a) Modelo de autorregistro de listado de actividades realizadas durante el día

\begin{tabular}{l} 
LISTADO DE ACTIVIDADES REALIZADAS DURANTE EL DIA \\
\hline "Estoy yendo al Centro Comercial solo y mirando las tiendas, \\
aunque no tengo la paciencia de ir de tienda en tienda y \\
pararme con demasiada tranquilidad, fijándome bien en las \\
cosas". \\
"He ido dos veces al cine solo como también he estado, antes \\
de ir a la película, tomando algunos caprichos". \\
- Tanto en las tiendas pero, sobre todo, cuando estoy sentado \\
lomándome algo en un bar, me siento incómodo porque creo \\
que se están lijando en mr".
\end{tabular}

b) Modelo de autorregistro de detección de distorsiones cognitivas

\begin{tabular}{|l|l|}
\hline PENSAMIENTOS NEGATIVOS & \multicolumn{1}{c|}{ DISTORSIONES } \\
\hline "Me voy a quedar sin habla el lunes & Anticipar negativamente, rumiaciones y \\
(bloqueado)". & magnificar negativamente. \\
- & Culpabilidad ("debería saber más y ser \\
situaciones durante el aprendizaje & más atrevido"), anticipar \\
negativamente ("mañana deseo hacer & algo distinto y no deseo fallar") y \\
(cambio de actividad, de compañeros, & minimizar lo positivo ("siempre creo \\
ambiente...) y no saber hacerlo y & que hago poco"). \\
quedarme solo". & \\
\end{tabular}


c) Modelo de autorregistro de evidencias

\begin{tabular}{|c|c|c|c|}
\hline $\begin{array}{l}\text { PENSAMIENTOS } \\
\text { NEGATIVOS }\end{array}$ & $\begin{array}{c}\text { EVIDENCIASA } \\
\text { FAVOR DE LOS } \\
\text { PENSAMIENTOS } \\
\text { NEGATIVOS }\end{array}$ & $\begin{array}{c}\text { EVIDENCIAS EN } \\
\text { CONTRA DE LOS } \\
\text { PENSAMIENTOS } \\
\text { NEGATIVOS }\end{array}$ & $\begin{array}{l}\text { PENSAMIENTOS } \\
\text { POSITIVOS }\end{array}$ \\
\hline $\begin{array}{l}\text { - Tengo } \\
\text { miedo de que } \\
\text { en el trabajo } \\
\text { me entiendan } \\
\text { bien y sepan } \\
\text { como soy } \\
\text { realmente. } \\
\text { Caer aburrido } \\
\text { y pesado son } \\
\text { mis historias } \\
\text { y traumas". }\end{array}$ & - Ninguna & $\begin{array}{l}\text { "Me estoy } \\
\text { anticipando". }\end{array}$ & $\begin{array}{l}\text { "Nunca sabré a } \\
\text { clencla clerta } \\
\text { cómo le caeré a } \\
\text { la gente porque } \\
\text { eso es algo } \\
\text { anecdótlco en } \\
\text { un trabajo con } \\
\text { muchas más } \\
\text { Importancla". }\end{array}$ \\
\hline
\end{tabular}

d) Modelo de autorregistro de reestructuración cognitiva

\begin{tabular}{|c|c|}
\hline PENSAMIENTOS NEGATIVOS & PENSAMIENTOS POSITIVOS \\
\hline $\begin{array}{l}\text { - "Soy incapaz de valerme por mí } \\
\text { mismo. No hago nada más que } \\
\text { quejarme y ver pasar la vida } \\
\text { alrededor mio, quedándome en } \\
\text { un segundo plano". } \\
\text { - "A la gente debo parecerle un } \\
\text { bicho raro y sienten pena por } \\
\text { me". }\end{array}$ & $\begin{array}{l}\text { - "Tengo demaslado miedo } \\
\text { pero debo adaptar el cuerpo } \\
\text { y la mente a la situaclón que } \\
\text { estoy viviendo". Debo } \\
\text { dejarme un poco de tlempo. } \\
\text { - No debo pensar en eso, son } \\
\text { cosas mías. Me observo } \\
\text { demasiado". }\end{array}$ \\
\hline
\end{tabular}

Una vez finalizado el tratamiento, se llevaron a cabo 10 sesiones de seguimiento. Durante estas sesiones se abordaron tres aspectos generales. (i) la continuación de la re-etiquetación de las creencias delirantes y la disminución de las conductas comprobatorias perfeccionando y potenciando el uso autorregistro en el manejo de la reestructuración cognitiva. (ii) El fomento de habilidades sociales en la solución de problemas y toma de decisiones. En este caso, se apoyó en un protocolo de autorregistro que permitía al paciente la identificación y la planificación del afrontamiento de situaciones sociales que percibía como conflictivas. El modelo de autorregistro se recoge en la tabla 4.

Finalmente (iii) se abordó la rehabilitación psicosocial proporcionándole al paciente la información necesaria de todos aquellos recursos disponibles en la sociedad para involucrarse en actividades 
Tabla 4.- Modelo de autorregistro de solución de problema y toma de decisiones

FASES DE LA AESOLUCION DE PROBLEMAS

1.- Orientación del problema ( ¿qué actitud he de tener ante los problemas?).

2.- Formulaclón y definlclón del problema (Lcuál es el problema?).

3.- Generación de alternativas (¿qué puedo hacer para soluclonarlo?).

4.- Toma de decislones (¿qué consecuenclas negativas y positivas me puede traer cada alternativa?).

5.- Ejecución y verlficación de resultados (llevo a cabo estas alternativas y observo si solucionamos realmente el problema si no es así, revisaré el proceso).

ALTERNATIVA:

\begin{tabular}{|c|c|}
\hline VENTAJAS (PARA MI) & INCONVENIENTES (PARA MI) \\
\hline & \\
\hline
\end{tabular}

lúdicas, recreativas y de formación (senderismo, deportes, cursos de formación...). Por último, durante la última sesión de seguimiento, se pasó de nuevo la batería de pruebas ya descritas.

\section{RESULTADOS}

Para evaluar los resultados se han tenido en cuenta tanto los datos derivados del autoanálisis y el autorregistro del paciente, como de las pruebas tipificadas administradas.

Comenzando con los datos referidos a los objetivos terapéuticos, los distintos autorregistros nos permitieron delimitar en qué medida esos objetivos se fueron cumpliendo, según la propia versión del paciente, tanto a nivel cognitivo como a nivel comportamental.

A nivel cognitivo, se ha tomado como ilustración de la evolución el número de pensamientos negativos referidos a la naturaleza del problema planteado (ideas delirantes de ser controlado: "los demás me están escuchando", "los demás me están mirando fijamente"; ideas delirantes persecutorias: "cuatro personas van insultándome y persiguiéndome", "cuatro coches me perseguian”, ideas delirantes de 
celos, ideas delirantes de transformación corporal: "me siento como irme", "he sentido la muerte cerca", alucinaciones auditivas como voces amenazantes: "oigo insultos, burlas, risas, besos, cuando voy caminando por la calle", "oigo cómo me tiran besos cada vez que me doy la vuelta en la calle"). En la figura 1 se resume su evolución, para aquellas sesiones en las que el paciente completó los autorregistros.

Figura 1.- Evolución de los pensamientos negativos, según los autorregistros del paciente

$\square$ pensamientos negativos

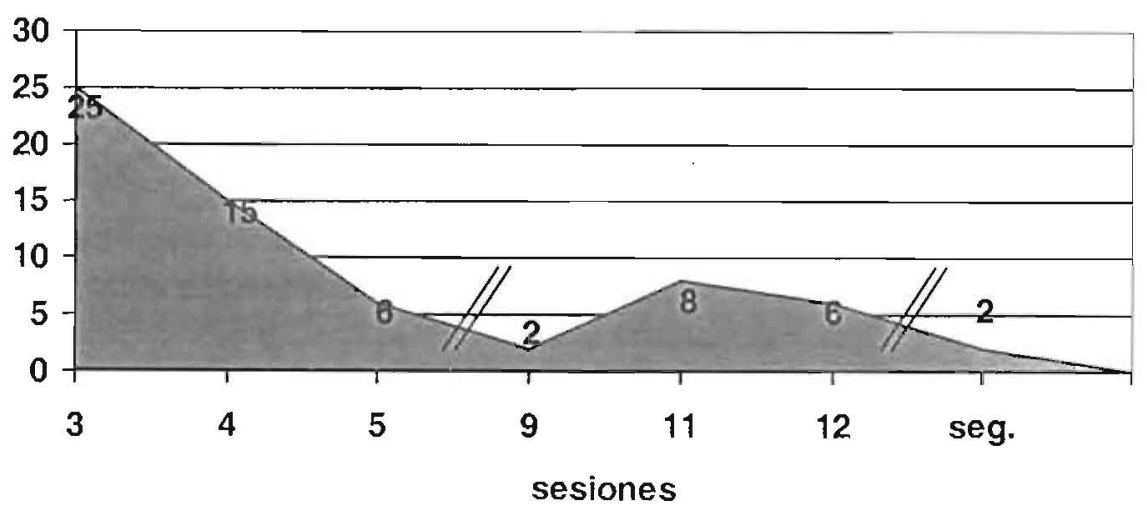

Como puede observarse, existe una clara línea descendente en la evolución de esos pensamientos que le perturbaban y que le hacian tener una idea distorsionada del contexto que le rodeaba (uno de los pensamientos que descendió de una manera significativa fue el relacionado con la creencia delirante de ir caminando por la calle y la gente se burlaba de él, aspecto que le preocupaba profundamente). La evolución, además ha sido clara. De los 25 pensamientos negativos registrados en la primera semana pasan a 15 en la segunda para mantenerse en un rango de 6 a 8, llegando incluso a sólo dos pensamientos distorsionados o disfuncionales.

Hay que señalar que esa disrninución de ideaciones negativas estaba directamente relacionada con el uso de estrategias que modificaban el modo que tenía de interpretar la realidad, por lo que esa disminución se 
vio acompañada de un incremento en pensamientos más racionales, lógicos y realistas.

A nivel comportamental se tuvo en cuenta para el análisis, la valoración que el paciente hacía de sus conductas de carácter negativo. De acuerdo con el análisis funcional, esas conductas se agrupaban en conductas de evitación y escape (constituidas por conjunto heterogéneo como evitación de relaciones interpersonales, dirigirse a alguien, equivocarse, contacto ocular, miedo a la oscuridad y a los insectos), y conductas de comprobación (básicamente relacionadas con sus ideas delirantes de persecución y control, comportamientos ritualistas en relación con las burlas y relacionados con la idea de muerte). El paciente era requerido por la terapeuta para que valorara su evolución en esas conductas. Posteriormente, la terapeuta valoraba esa evolución de acuerdo con las siguientes categorías: (3) no cambio, que indicaba que las conductas no habian evolucionado; (2) cambio mínimo, cuando las conductas habían disminuido, pero no de manera satisfactoria para el paciente; (1) reducción o mejora, cuando las conductas habian disminuido, pero ahora valoraba esa disminución como satisfactoria; y (0) eliminación, cuando el paciente indicaba que esas conductas ya no se presentaban. En la figura 2 se muestra la evolución de esos grupos de conductas, en base a la categorización anterior.

Figura 2.- Evolución de los comportamiento negativos (evitación y escape, y conductas de autocomprobación), según los autorregistros del paciente

\section{Q evitación y escape 貝 comprobación}

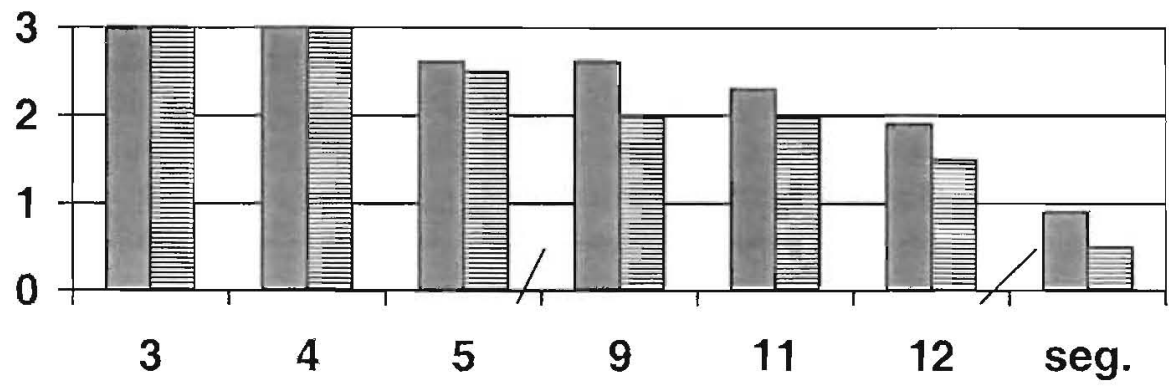

sesiones 
De nuevo, se puede constatar una clara tendencia a la disminución de los comportamientos negativos. De acuerdo con las categorías utilizadas, se puede observar una tendencia desde las sesiones iniciales donde no se produclan cambios (inicio del trabajo terapéutico) a unos cambios mínimos en las sesiones posteriores. Una vez finalizadas las sesiones de tratamiento se pudo constatar una disminución significativa, considerada satisfactoria por el paciente. Gran parte de las conductas recogidas en las clases de conducta de evitación y escape, y de comprobación llegaron a la categoría de cero en las últimas sesiones y en el seguimiento (eliminadas). Algunas conductas, sin embargo (p.e., el miedo a equivocarse), no tuvieron cambios apreciables.

Por lo que se refiere a la evolución detectada en las pruebas tipificadas administradas, se han tomado los datos referidos a las primeras sesiones de evaluación, al final del tratamiento y en el seguimiento.

Presentamos en primer lugar los datos referidos a los componentes psicopatológicos y, en un segundo momento, los datos referidos a las relaciones sociales e interpersonales. En este sentido, en la tabla 5 se recogen los datos detectados por las PANSS y por el Inventario de Depresión de Beck.

Tabla 5.- Percentiles obtenidos por el paciente en la escala PANSS y puntuaciones directas en el BDI en tres periodos de evaluación (pre, post y seguimiento) para tres de las escalas

\begin{tabular}{|l|c|c|c|}
\hline & Pretratamiento & Postratamiento & Seguimiento \\
\hline PANSS Sintomas positivos & 50 & 25 & 5 \\
\hline PANSS SIntomas negativos & 25 & 25 & 5 \\
\hline PANSS Síntomas generales & 50 & 25 & 5 \\
\hline BDI & 37 & 27 & 16 \\
\hline
\end{tabular}

De nuevo, se observa una clara tendencia a la disminución de la sintomatología psicopatológica, especialmente en los síntomas generales (concretamente y de manera más llamativa, los relacionados con la ansiedad, sentimientos de culpa, depresión, desorientación, atención, control de irnpulsos y evitación social activa) y en los síntomas positivos. En el caso de los síntomas negativos, hay que indicar que el paciente no había empezado a desarrollar muchos de esos síntomas, por lo que su disminución es menos pronunciada. Dado que se dispone de las puntuaciones en percentiles, la evolución detectada puede 
considerarse clínicamente significativa. La disminución pre-post es de un $50 \%$ para la sintomatología general y positiva. Cuando examinamos los datos del seguimiento esa caída es mucho más evidente porque el percentil en las tres escalas es de 5 .

Si tomamos en cuenta los datos sobre la sintomatología depresiva, el paciente partía de un nivel grave (una puntuación directa de 37). A pesar de que no se trabajó directamente sobre los pensamientos depresogénicos, los datos del postratamiento indican un paso de un nivel grave a un nivel de depresión moderado. Este nivel alcanza a un nivel leve en el seguimiento. En este sentido, comparando los datos con el nivel inicial, el porcentaje de disminución de la sintomatología es pequeño en el postratamiento (sobre un $20 \%$ ), pero es clínicamente apreciable en el seguimiento (disminución de un $56 \%$ ).

Con respecto la mundo social y de relaciones interpersonales, una de las fuentes de preocupación y temor del paciente, y tomando en cuenta los datos provenientes del cuestionario de agorafobia, en la tabla 6 se resumen los datos para los tres momentos evaluativos y para las tres escalas del cuestionario.

Tabla 6.- Puntuaciones directas del paciente en el cuestionario de agorafobla en las tres subescalas (motora, cognitiva y fisiológica), para los tres momentos evaluativos

\begin{tabular}{|l|c|c|c|}
\hline \multicolumn{1}{|c|}{ Subescalas } & Pre & Post & Seguimiento \\
\hline Conducta motora & 200 & 174 & 103 \\
\hline Cognitiva & 51 & 28 & 16 \\
\hline Fisiológica & 90 & 75 & 60 \\
\hline
\end{tabular}

Los datos de partida, como se indicó anteriormente, eran indicativos de un alto nivel de impedimento en sus relaciones sociales e interpersonales. Su alto grado de temor a moverse con cierta libertad y comodidad se observa en el elevado grado de comportamientos cotidianos (dentro del bloque de las conductas motoras de evitación / escape señalar su temor por: la posibilidad del establecimiento de relaciones interpersonales, la resolución de problemas, hablar en público, equivocarse o fracasar, mirar a la gente directamente a la cara, salir por los alrededores de su casa y la oscuridad y dentro a su vez, del bloque de las conductas de comprobación señalar: la presencia 
pronunciada de ideas delirantes relacionadas con la posibilidad de morir y persecutorias), que le generan ansiedad. Las subescalas cognitivas y fisiológicas también muestran un nivel de partida de un intenso grado de malestar.

La evolución en este caso, siendo positiva, no es tan remarcable como en los datos anteriores. Así, el componente motor muestra una disminución importante, pero todavía se mantiene a un nivel clínicamente apreciable (incluso los datos más positivos del seguimiento, no permiten indicar una evolución clínicamente significativa). Algo parecido ocurre con la activación fisiológica negativa: aunque disminuye, no lo hace de manera clínicamente apreciable. Sin embargo, en la subescala cognitiva ("Tengo demasiado miedo pero debo adaptar el cuerpo y la mente a la situación que estoy viviendo". "Debo dejarme un poco de tiempo". "No debo pensar en eso, son cosas mías. Me observo demasiado"), los datos sí son apreciables, disminuyendo los pensamientos inadecuados hasta un $70 \%$. Posiblemente, el hecho de que el programa se centrara en las cogniciones disfuncionales en relación con la sintomatología positiva del trastorno, pudo conllevar un efecto de derivación hacia este tipo de pensamientos agorafóbicos.

Sin embargo, si examinamos sus habilidades sociales podemos observar algunas matizaciones con respecto al temor y ansiedad que le provoca el mundo social. En la figura 3 representamos la evolución de las habilidades sociales medidas por el cuestionario de HHSS, agrupadas en las cuatro categorías de respuesta, en los tres momentos evaluativos.

Analizando cada momento evaluativo, en un principio el paciente relata que no es capaz de llevar a cabo ninguno de los comportamientos requeridos en el cuestionario, siendo lo más frecuente la inhibición comportamental. Una vez finalizada la intervención, el paciente es capaz de llevar a cabo algunos comportamientos sociales (solicitar información, expresar afecto o elogio, hacer una caricia-broma, recibir afecto, admiración, aceptar el contacto físico), pero también aumentan ahora las conductas que maneja de manera inadecuada. En síntesis, las conductas que le generaban ansiedad o las que prefería evitar pasaron de 17 en la fase inicial, a 7 en el postratamiento. De las 10 condiciones restantes, 5 señala que es capaz de desarrollar un comportamiento hábil y 5 considera que no posee las habilidades necesarias para 
Figura 3.- Habilidades sociales (cuestionario de HH.SS.) del paciente, agrupadas por el tipo de respuesta ante situaciones sociales, en los tres momentos evaluativos

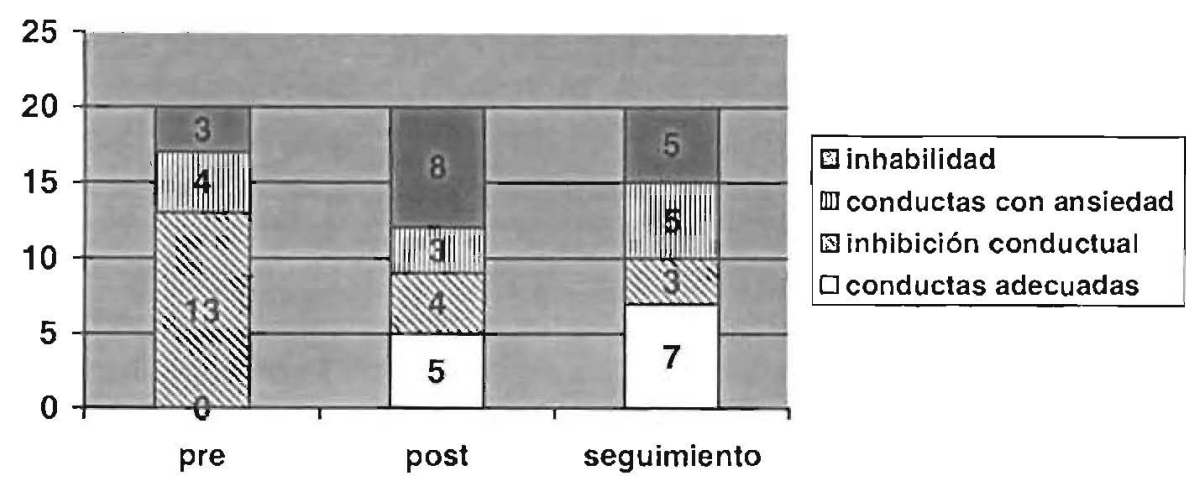

abordarlas. En el seguimiento los datos se mantienen en general o mejoran, destacando cómo algunas condiciones identificadas previamente como 'inhabilidad' ahora considera que es capaz de llevarlas a cabo adecuadamente.

Si contrastamos estos datos con los de agorafobia, podemos reconocer que el paciente continúa con un temor apreciable a las relaciones sociales e interpersonales, especialmente en los niveles comportamentales y fisiológicos, pero también se puede constatar que se ha ido produciendo una mejoría en la medida en que las situaciones sociales que le producian ansiedad o trataba de evitarlas o se inhibia, se han ido cambiando hacia una mayor capacidad de afrontamiento por parte de paciente. La evolución en las habilidades sociales se puede considerar clínicamente significativa en la medida en que los comportamientos hábiles pasaron de cero a 7 en el seguimiento y el número de condiciones que inhibian o generaban ansiedad disminuyeron más de un $50 \%$. 
En resumen, de acuerdo con los datos presentados, se observa una evolución favorable del paciente. Esta evolución es perceptible en la disminución apreciable de sus pensamientos distorsionados (especialmente, las relacionadas con el bloque de conductas comprobatorias relacionadas con las ideas delirantes de muerte y persecución como por ejemplo: llevar gafas puestas e ir caminando por la calle mirando al suelo constantemente), vinculados a la sintomatología positiva. Como producto de ello esa sintomatología ha disminuido, así como los síntomas psicopatológicos generales. El nivel de depresión actual es leve y sus temores y miedos sociales han disminuido, capacitando al paciente para llevar a cabo una serie de comportamientos en sus relaciones interpersonales de manera satisfactoria.

\section{DISCUSIÓN}

Hemos presentado el abordaje terapéutico de un paciente diagnosticado con esquizofrenia paranoide en el que en él asumió la dirección del proceso de cambio. Desde el primer momento de la valoración del caso se pudo observar que el paciente poseía una capacidad intelectiva adecuada, una actitud positiva para el cambio (estaba preparado para cambiar), preponderancia de sintomatología positiva y elevada conciencia de enfermedad. Además, la propia naturaleza del problema (con niveles elevados de suspicacia), hizo pensar en la existencia de elevados niveles de reactancia psicológica, lo que podía hacer peligrar un proceso terapéutico 'demasiado' dirigido por la terapeuta y que el paciente lo viera como una limitación de su libertad o una manipulación. Todo ello justificó el que se potenciara en el paciente un papel activo en la terapia, favoreciendo el role de "actor" en su proceso de cambio. Evidentemente, si estas características del paciente no hubieran estado presentes, la autorregulación no habría estado justificada.

La autodirección estuvo constituida, básicamente, en la elaboración y seguimiento conjunto (terapeuta-paciente) de un modelo de autorregistro que potenciara por un lado, la motivación y adherencia al tratamiento y la reducción de la posible reactancia que pudiera aparecer a la hora de la reelaboración de sus creencias delirantes. El tratamiento constó de las siguientes fases: evaluación, tanto cualitativa como 
cuantitativa del caso, psicoeducación familiar, reestructuración cognitiva (de forma activa), exposición en vivo a estímulos temidos y adquisición de habilidades sociales y de resolución de problemas. Tras la evaluación post-tratamiento y el seguimiento, se apreciaron una mejorias clínicamente significativas en la mayor parte de las áreas evaluadas inicialmente, apreciando al mismo tiempo, un incremento progresivo en la percepción de las mejorias alcanzadas por parte del paciente. Estas mejoras implicaron un aumento paulatino en su nivel de actividad, en general, y más específicamente, en tomar él mismo la iniciativa en la organización de actividades lúdicas (excursiones de senderismo, petición de un bono-bus para el conocimiento de la isla, entrenar a un equipo de fútbol femenino), con el grupo de compañeros del curso de contabilidad que estaba realizando en ese momento. Este hecho a su vez mejoró el objetivo de fomentar sus habilidades sociales y establecer nuevas relaciones interpersonales.

También se puede comentar, como se aprecia en la figura 1, la existencia de una disminución clara de los pensamientos negativos que presentaba el paciente y que le generaban una elevado grado de malestar y limitación a la hora de afrontar las diferentes situaciones de su vida cotidiana debido a la visión distorsionada que presentaba de su entorno sobresaltando la reducción pronunciada de la creencia delirante de ir caminando por la calle y creer que le tiraban besos, le perseguian y se burlaban de él. Dicha reducción de ideaciones negativas estaba directamente relacionada con el aprendizaje, empleo y profundización de la reestructuración cognitiva con el objetivo de minimizar tales pensamientos negativos y potenciar la adquisición y afianzamiento de pensamientos alternativos más adaptativos y por consiguiente, más racionales, lógicos y sobre todo, realistas. Como ejemplos de estos últimos, citamos los siguientes: "No debo pensar en eso, son cosas mías, me observo demasiado", "no debo juzgar las situaciones, sino observarlas y experimentar las sensaciones sin ningún tipo de miedo. No te juzgues, sólo observa", "Lo importante es ser persona y no perder la cabeza, o sea, agarrarte fijamente a tus principios","aunque no tengas nada, una persona debe tener unos motivos por los que luchar", "tengo gente a mi alrededor y debo darme cuenta de ello". Como consecuencia de la mejoría destacada en el manejo y reelaboración de las creencias delirantes del paciente, se 
produjo una mejoría pronunciada en los niveles de depresión iniciales dado el efecto de generalización de dicho control. Asimismo, se produjo una reducción en el componente cognitivo de sus preocupaciones agorafóbicas, favoreciendo con ello el afrontamiento a las situaciones temidas, especialmente las relacionadas con el establecimiento de relaciones interpersonales. Todo ello permite apreciar que las mejoras alcanzadas son más pronunciadas en el componente cognitivo.

De manera general, se puede decir que se han visto cumplidos los objetivos terapéuticos, resaltando especialmente, los relacionados con la reelaboración de las creencias delirantes y pensamientos negativos que se han reducido de una manera clínicamente significativa, llegando el propio paciente a verbalizar la no existencia de las mismas o en todo caso, si tales creencias aparecieran él mismo las daba por aceptadas y se comprometía a ignorarlas ya que "no tenían necesariamente que ver con él". Con ello, se plantea una forma alternativa de enfoque terapéutico, para los casos que presenten las características anteriormente mencionadas, que potencia el control de la sintomatología negativa y la reducción de la sintomatología positiva.

Pero, a pesar de los resultados positivos extraídos de la aplicación del tratamiento elaborado y presentado en este artículo, hay que señalar uno de los puntos más críticos en el abordaje terapéutico: la irregularidad en la cumplimentación de los modelos de autorregistro. Siendo un elemento esencial en la motivación y adherencia al tratamiento, en ocasiones no fue cumplimentado adecuadamente. Esto nos plantea, por un lado, la necesidad de fortalecer constantemente la utilidad del binomio autorregistro-autorregulación, y, por otro, la necesidad de elaborar protocolos que no le supongan un esfuerzo considerable la paciente, aunque ello implique pérdida de información sobre algunos contenidos.

Otro aspecto crítico fue la escasa asistencia de los familiares al requerimiento de la terapeuta. La colaboración e implicación de los familiares favorece no sólo la adherencia del paciente al tratamiento sino, y de manera importante, el afrontamiento positivo y adaptativo por parte de los familiares a la situación de convivir con un familiar con esquizofrenia. Está constatado que el clima familiar es un elemento importante en la reducción de las recaldas y un factor de protección, tanto para el paciente como para los familiares que lo 
toman a cargo. Sin embargo, no se pudo implicar a los padres más allá de unas sesiones de psicoeducación.

Quisiéramos acabar comentando algunos aspectos incidentales, pero que consideramos importantes: Durante el seguimiento (que se le sigue realizando en la actualidad) el paciente consiguió un contrato laboral (IFEM) de cuatro meses de duración, como contable, tras haber realizado un curso de contabilidad y administración (SIMPROMI), en el que alcanzó la máxima calificación. En la actualidad ha sido contratado, de forma indefinida, en una empresa de publicidad como uno de los contables de la misma, cumpliendo con su horario de trabajo normal.

En definitiva, se puede concluir que la cronicidad de un trastomo como el de la esquizofrenia puede ser aminorada y controlada si se logra actuar a tiempo y de una manera adecuada y sistematizada, si están adecuadamente medicados, y si el paciente presenta una buena conciencia del trastorno que padece y una disposición activa para el cambio.

\section{REFERENCIAS BIBLIOGRÁFICAS}

Anderson, C.M., Reiss, D.J. y Hogarty, G.E. (1986). Schizophrenia in the family: A Practitioner's Guide to Psychoeducation and Management. Nueva York: The Guildford Press.

Anthony, W. y Liberman, R.P. (1986). The practice of psychiatric rehabilitation: Historial, conceptual, and research base. Schizophrenia Bulletin, 12, 542-559 (número especial).

Barrowclough, C. y Tarrier, N. (1992). Families of schizophrenic patients:

Cognitive behavioural intervention. Londres: Chapman y Hall.

Beck, A.T., Ward, C.H., Mendelson, M., Mock, J. y Erbaugh, J. (1961).

An inventory for measuring depression. Archives of General Psychiatry, 4, 561-571.

Bellack, A.S., Morrison, R.L. y Mueser, K.T. (1989). Social problem solving in schizophrenia. Schizophrenia Bulletin, 15, 101-116.

Benton, M.K. y Schoroeder, H.E. (1990). Social skills training with schizophrenics: A meta-analytic evaluation. Journal of Consulting and Clinical Psychology, 58, 741-747.

Birchwood, M.J., Hallett, S.E. y Preston, M.C. (1989). Schizophrenia: An integrated approach to research and treatment. Nueva York: New York University Press. 
Brenner, H.D., Hirsbrunner, A. y Heimberg, D. (1996). Integrated psychological therapy program: Training in cognitive and social skills for schizophrenic patients. En P.W. Corrigan y S.C. Yudofsky (Eds.). Cognitive rehabilitation for neuropsychiatric disorders (pp. 329-348). Washinnton, DC: American Psychiatric Association.

Brenner, H.D., Hodel, B., Roder, V. y Corrigan, P. (1992). Integrated psychological therapy for schizophrenic patients (IPT): Basic assumptions, current status and future directions. En: F.P. Ferrero, A.E. Haynal y N. Sartorious (Eds.) Schizophrenia and affective psychoses: Nosology in contemporary psychiatry (pp 201-209). London: Jonhn Libbey.

Camisa, K.M., Bockbrader, M.A. y Lysaker, P. (2005). Personality traits in schizophrenia and related personality disorders. Psychiatry Research, 133, 23-33.

Chambon, O., Marie-Cardine, M. y Dazord, A. (1996). Social skills training for chronic psychotic patients: A Frehch study. European Psychiatry, 11, 77-84.

Corrigan, P.W. (1991). Social skills training in adult psychiatric populations: A meta-analysis. Journal of Behaviour Therapy and Experimental Psychiatry, 22, 203-210.

Drury, V., Birchwood, M. y Cochrane, R. (1996). Cognitive therapy and recovery from acute psychosis: A controlled trial: I. Impact on psychotic symptoms. British Journal of Psychiatry, 169, 593-601.

Drury, V., Birchwood, M. y Cochrane, R. (1996). Cognitive therapy and recovery from acute psychosis: A controlled trial: II. Impact on recovery time. British Journal of Psychiatry, 169, 602-607.

Echeburúa, E., Corral, P., Garcia, E., Paez, D. y Borda, M. (1992). Un nuevo inventario de agorafobia (IA). Análisis y Modificación de Conducta, 18, 101-123.

Falloon, I., Laporta, M. y Fadden, G (1993). Managing stress in families: Cognitive and behavioural strategies of enhancing coping skills. Florence, Kentucky: Taylor y Frances/Routledge.

Freeman, D., Garety, P.A. y Kuipers, E. (2002). A cognitive model of persecutory delusions. British Joumal of Clinical Psychology, 41, 331-347. Garety, P., Fowler, D. y Kuipers, E. (2000). Cognitive-behavioural for medication-resistant symptoms. Schizophrenia Bulletin, 26, 73-86 (número especial). 
Goldstein, M.J. (1994). Psychoeducational and family therapy in relapse prevention. Acta Psiquiatrica Scandinavica, 28, 54-57.

Goldstein, M.J., Rodnick, E.H., Evans, J.R., Philip, R.A., May, R.A. y

Steinberg, M.R. (1978). Drug and family therapy in the aftercare of acute schizophrenic. Archives of General Psychiatry, 35, 1169-1177. Haddock, G y Tarrier, N. (1998). Assessment and formulation in the cognitive behavioural treatment of psychosis. En N. Tarrier, A. Wells y G. Haddock (Eds.). Treating complex cases: The cognitive behavioural therapy approach (pp. 155-175). Nueva York: Wiley.

Haddock, G, Sellwood, W. y Tarrier, N. (1994). Developments in cognitive-behaviour therapy for persistent psychotic symptoms. Behaviour Change, 11, 200-212. (número especial).

Haddock, G, Tarrier, N. y Morrison, A.P. (1999). A pilot study evaluating the effectiveness of individual inpatient cognitive-behavioural therapy in early psychosis. Social Psychiatry and Psychiatric Epidemiology, 34, 254-258.

Hartman, L.M. y Cashman, F.E. (1983). Cognitive-behavioral and psychopharmacological treatment of delusional symptoms: a preliminary report. Behav Psychoter, 11, 50-61.

Heissen, R.K., Liberman, R.P.y Kopelowicz, A. (2000). Psychosocial skills training for schizophrenia: Lessons from the laboratory. Schizophrenia Bulletin, 26, 21-46 (número especial).

Jakes, S., Rhodes, J. y Turner, T. (1999). Effectiveness of cognitive therapy for delusions in routine clinical practice. British Journal of psychiatry, 175, 331-335.

Jolley, S., Garety, P.y Craig, T. (2003). Cognitive Therapy in Early

Psychosis: A Pilot Randomized Controlled Trial. Behavioural and Cognitive Psychotherapy, 31, 473-478.

Joyce, J., Leese, M. y Kuipers, E. (2003). Evaluating a model of caregiving for people with psychosis. Social Psychiatry and Psychiatric Epidemiology, 38, 189-195.

Kay, S.R., Fiszbein, S. y Opler, L.A. (1987). The Positive and Negative Sindrome Scale for Schizophrenia. Schizophrenia Bulletin, 13, 261276.

Kemp, A. y David, A. (1996). Psychological predictors of insight and compliance in psychotic patients. British Journal of Psychiatry, 169, 444-450. 
Kemp, R., David, A. y Hayward, P. (1996). Compliance therapy: An intervention targeting insight and treatment adherence in psychotic patients. Behavioural and Cognitive Psychotherapy, 24, 331-350.

Kemp, R., Kirov, G y Everit, B. (1998). Randomised controlled trial of compliance therapy: 18-month follow-up. British Journal of Psychiatry, 172, 413-419.

Kingdon, D. (2004). Cognitive-behavioural therapy for psychosis. British Journal of Psychiatry, 184, 85-86.

Kingdon, D. G. y Turkington, D. (2005). Cognitive therapy of schizophrenia. Nueva York: Guilford Press.

Kingdon, D.G y Turkington, D. (1991). The use cognitive behaviour therapy with normalizing rationale in schizophrenia: Preliminary report. Journal of Nervous and Mental Disease, 179, 207-211.

Kuipers, E. (2005). Evaluating Cognitive Behavior Therapy for Psychosis. Clinical Psychology: Science and Practice, 12, 65-67. Kuipers, E., Garety, P. y Fowler, D. (1997). London-East Anglia randomised controlled trial of cognitive-behavioural therapy for psychosis, I: Effects of the treatment phase. British Journal of Psychiatry, 171, 319-327.

Kuipers, E., Leff, J.P. y Lam, D. (1992). Family work for schizophrenia: A practical guide. London: Gaskell.

Leff, J.P., Kuipers, L., Berkowitz, R. y Sturgeon, D. (1985). A controlled trial of social intervention in the families of schizophrenic patients: two year follow-up. British Journal of Psychiatry 146, 594-600.

Lehman, A.F., Carpenter, W.T., Goldman, H.H. y Steinwachs, D.M. (1995).Treatment outcomes in schizophrenia: Implications for practice, policy, and research. Schizphrenia Bulletin, 21, 669-675.

Liberman, R.P. (1986). Coping and Competence as protective factors in the vulnerability-stress model of schizophrenia. En M.J. Goldstein, I. Hand y K. Hahlweg (Eds.) Treatment of schizophrenia: family assessment and intervention (pp. 201-215). Berlin: Springer-Verlag. Liberman, R.P. (1986). Psychiatric Rehabilitation of Schizophrenia: Editor's Introduction. Schizophrenia Bulletin, 12, 540-541.

Liberman, R.P. (Ed.). (1993). Rehabilitación integral del enfermo mental crónico. Barcelona: Martínez Roca.

Liberman, R.P., Vaccaro, J.V. y Corrigan, P.W. (1995). Psychiatric rehabilitation. En H.I. Kaplan y B.J. Sadock (Eds.) Comprehensive 
textbook of psichiatry/VI (pp.2696-2719). Baltimore, MD: Williams y Wilkins.

Norman, R.M.G y Townsend, L.A. (1999). Cognitive-behavioural therapy for psicosis: a status report. Canadian Journal Psychiatry, 44, 245252.

Nuechterlein, K.H., Dawson, M.E., Gitlin, M., Ventura, J., Goldstein, M.J., Snyder, K.S., et al. (1992). Developmental processes in schizophrenic disorders: Longitudinal studies of vulnerability and stress. Schizophrenia Bulletin, 18, 387-425.

Penn, D.L. y Mueser, K.T. (1996). Research update on the psychosocial treatment of schizophrenia. American Journal of Psychiatry, 153, 607-617.

Perris, C., Toresson, P., Skgerlind, L., Warburton, E., Gustavsson, H. y Johansson, T. (1990). Integrating components in a comprehensive cognitive treatment program for patients with a schizophrenic disorder. En C.N. Stefanis, A.D. Rabavilas y C.R. Soldatos (Eds.). Psychiatry: A world perspective (Vol., 3, pp. 724-729). Amsterdam: Elsevier.

Pilling, S., Bebbington, P. y Kuipers, E. (2002). Psychological treatments in schizophrenia: I. Meta-analysis of family intervention and cognitive behaviour therapy. Psychological Medicine, 32, 763-782.

Pilling, S., Bebbington, P. y Kuipers, E. (2002). Psychological treatments in schizophrenia: II. Meta-analyses of randomized controlled trials of social skills training and cognitive remediation. Psychological Medicine, 32, 783-791.

Rathod, S., Kingdon, D. y Smith, P. (2005). Insight into schizophrenia: The effects of cognitive behavioural therapy on the components of insight and association with sociodemographics-data on a previously published randomised controlled trial. Schizophrenia Research, 74, 211-219.

Schooler, N.R.y Keith, S.J. (1993). The clinical research base for the treatment of schizophrenia. Psychopharmacology Bulletin, 29, 431446.

Segura, M. (1981). Cuestionario de habilidades sociales. En: Ficha de Evaluación Conductual, ponencia libre presentada en el Primer Congreso de la AETCO. (Congreso Fundacional de la Asociación Española de Terapia de Comportamiento). Málaga, 29 de Abril a 2 de Mayo. 
Slade, M., Holloway, F.y Kuipers, E. (2003). Skills development and family interventions in an early psychosis service. Journal of Mental Health, 12, 405-415.

Smith, B. (2005). Cognitive Therapy of Schizophrenia. Primary Care \& Community Psychiatry. The international journal for the management and treatment of mental health problems in primary care and the community, 10, 78-79.

Stevens, S. y Sin, J. (2005). Implementing a self-management model of relapse prevention for psychosis into routine clinical practice. Joumal of Psychiatric and Mental Health Nursing, 12, 495501.

Stolar, N. (2005). The Case Study Guide to Cognitive Behaviour Therapy of Psychosis. Journal of Cognitive Psychotherapy, 19, 394-395.

Szmukler, G., Kuipers, E. y Joyce, J. (2003). An exploratory randomised controlled trial of a support programme for carers of patients with a psychosis. Social Psychiatry and Psychiatric Epidemiology, 38, 411-418.

Tarrier, N. y Barrowclough, C. (1995). Family interventions in schizophrenia and their long-term outcomes. International Journal of Mental Health, 24, 38-53.

Tarrier, N., Barrowclough, C. y Haddock, G (1999). The dissemination of innovative cognitive-behavioural psychosocial treatments for schizophrenia. Journal of Mental Health, 8, 569-582.

Tarrier, N., Beckett, R. y Harwood, S. (1993). A trial of two cognitivebehavioural methods of treating drug-resistant residual psychotic symptoms in schizophrenic patients: I. Outcome. British Journal of Psychiatry, 162, 524-532.

Tarrier, N., Beckett, R. y Harwood, S. (1993). A trial two cognitivebehavioral methods of treating drug-resistant residual psychotic symptoms in schizophrenic patients: I. Outcome. British Journal of Psychiatry, 162, 524-532.

Tarrier, N., Kinney, C.y McCarthy, E. (2001). Are some types of psychotic symptoms more responsive to cognitive-behavior therapy? Behavioural and Cognitive Psychotherapy, 29, 45-55.

Tarrier, N., Sharpe, L. y Beckett, R. (1993). A trial of two cognitive behavioural methods of treating drug-resistant residual psychotic 
symptoms in schizophrenic patients: Il. Treatment-specific changes in coping and problem-solving skills. Social Psychiatry and Psychiatric Epidemiology, 28, 5-10.

Wykes, T., Tarrier, N. y Lewis, S. (1998). Outcome and innovation in psychological treatment of schizophrenia. Nueva Jersey: Wiley.

Yusupoff, L. y Haddock, G. (1998). Options and clinical decision making in the assessment and psychological treatment of persistent hallucinations and delusions. En C. Perris y P.D. McGorry (Eds.). Cognitive psychotherapy of psychotic and personality disorders: Handbook of theory and practice (pp. 111-127). Nueva York: Wiley. Yusupoff, L. y Tarrier, N. (1996). Doping strategy enhancement for persistent hallucinations and delusions. En: G Haddock y P.D. Slade (Eds.) Cognitive - behavioural interventions with psychotic disorders (pp. 86-102). London: Routledge.

Zubin, J. y Spring, B. (1977). Vulnerability: A new view of schizophrenia. Journal of Abnormal Psychology, 86, 103-126. 